\title{
Enhancing of nonlinear thermoelectric response of a correlated quantum dot in the Kondo regime by asymmetrically coupling to the leads
}

\author{
D. Pérez Daroca, ${ }^{1,2}$ P. Roura-Bas, ${ }^{3,2}$ and A. A. Aligia ${ }^{4,2}$ \\ ${ }^{1}$ Gerencia de Investigación y Aplicaciones, Comisión Nacional de \\ Energía Atómica, (1650) San Martín, Buenos Aires, Argentina \\ ${ }^{2}$ Consejo Nacional de Investigaciones Científicas y Técnicas, (1025) CABA, Argentina \\ ${ }^{3}$ Centro Atómico Bariloche, Comisión Nacional de Energía Atómica, 8400 Bariloche, Argentina \\ ${ }^{4}$ Centro Atómico Bariloche and Instituto Balseiro, \\ Comisión Nacional de Energía Atómica, 8400 Bariloche, Argentina
}

\begin{abstract}
We study the low temperature properties of the differential response of the current to a temperature gradient at finite voltage in a single level quantum dot including electron-electron interaction, non-symmetric couplings to the leads and non-linear effects. The calculated response is significantly enhanced in setups with large asymmetries between the tunnel couplings. In the investigated range of voltages and temperatures with corresponding energies up to several times the Kondo energy scale, the maximum response is enhanced nearly an order of magnitude with respect to symmetric coupling to the leads.
\end{abstract}

PACS numbers: 73.23.-b, 71.10.Hf, 75.20.Hr

\section{INTRODUCTION}

During the last decade the study of nanodevices that convert heat into work has received great attention due to possible applications 1 . These quantum thermoelectric machines are usually considered to be composed by a system of a small number of degrees of freedom coupled to a set of macroscopic reservoirs. Among them, semiconducting ${ }^{2-\frac{8}{-8}}$ and molecular ${ }^{-1}-18$ quantum dots (QDs) play a fundamental role.

A permanent interest of enhancing efficiency and power of such devices exists. In this sense, the charge and heat currents through QDs have been focused of intense research both experimentally $\underline{\underline{19}} \underline{\underline{22}}$ and theoretically $\underline{\underline{19}, 23} \underline{\underline{-40}}$.

Mahan and Sofo have shown that a delta shape of the transmission function maximizes the efficiency of thermoelectric devices 23 . Since then, other mechanisms for increasing the Seebeck coefficient (thermopower), $S$, have been proposed. Among them, a time-dependent gate voltage ${ }^{30}$, orbital degeneracy ${ }^{31.32}$, negative values of the Coulomb repulsion 29 , quantum Hall bar with fractional filling factors $\stackrel{40}{ }$, and nonlinear transport effects $27,35,39$ have been investigated.

Recently, Dorda et al. studied the differential thermoelectric response of a correlated impurity in the nonequilibrium Kondo regime at finite voltage ${ }^{35}$ In addition to providing a fundamental understanding of the system, the authors point out the potential of quantum dots as possible nanoscale temperature sensors. The study was limited to symmetric coupling of the dot to both leads, and the authors state that further studies including asymmetric couplings to the leads are required to fully assess the potential of quantum dot devices for nanoscale sensing applications.

In this work we consider a QD asymmetrically coupled to the leads and we show that for large asymmetry, which is expected for molecular $\mathrm{QD}^{41,42}$, the differential

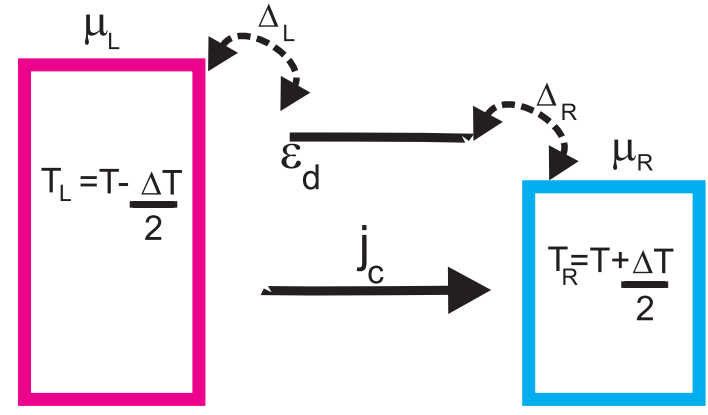

FIG. 1. (Color online) Scheme of the model considered, with the electronic level of the QD and their hybridization to the leads.

response of the current to a temperature gradient at finite voltage is increased nearly by an order of magnitude with respect to the symmetric case.

Specifically, we investigate nonlinear (NL) transport effects on the differential thermopower. When a bias voltage is applied to the system a constant electric current in the steady state is established. Assuming this regime, we analyzed its response to an infinitesimal gradient of temperature between the leads, as sketched in Fig. 1. We restrict our study to the case in which transport is due to electrons. Vibrational effects were studied in Refs. 22, 27, and 37, among others. The model is suitable for molecular QDs (for which asymmetric couplings are the most usual situation) and semiconducting QDs in which the tunnel couplings can be tuned at will ${ }^{8}$. We find that the asymmetry of the tunneling couplings, $\alpha=\Delta_{L} / \Delta_{R}$, plays a nontrivial role and large values of $\alpha$ boost the differential thermopower in the NL regime. As it will be clarified below, the range of parameters for this enhancement are within or near the Kondo regime. 


\section{MODEL AND EXPRESSION FOR THE CURRENT}

We employ the single impurity Anderson Hamiltonian to model the molecular or semiconducting QD. It is composed by a single dot level of energy $E_{d}$ connected to two metallic reservoirs. The Hamiltonian is

$$
\begin{aligned}
H= & E_{d} n_{d}+U n_{d \uparrow} n_{d \downarrow}+\sum_{\nu k \sigma} \epsilon_{k}^{\nu} c_{\nu k \sigma}^{\dagger} c_{\nu k \sigma} \\
& +\sum_{\nu k \sigma}\left(V_{k}^{\nu} d_{\sigma}^{\dagger} c_{\nu k \sigma}+\text { H.c. }\right)
\end{aligned}
$$

where $n_{d}=\sum_{\sigma} n_{d \sigma}, n_{d \sigma}=d_{\sigma}^{\dagger} d_{\sigma}, d_{\sigma}^{\dagger}$ creates an electron with spin $\sigma$ at the active level of the QD, $c_{\nu k \sigma}^{\dagger}$ creates a conduction electron at the left $(\nu=L)$ or right $(\nu=R)$ lead, and $V_{k}^{\nu}$ describe the hopping elements between the leads and the QD.

When the temperatures $T_{\nu}$ and/or chemical potentials $\mu_{\nu}$ of the reservoirs are different, heat and electric (charge) currents flow from one lead to the other. The sign of such currents depend on the temperature and chemical potentials differences and they are constant in the steady state. As a reference, we assume $T_{\nu}=T+\gamma_{\nu} \Delta T / 2$ and $\mu_{\nu}=-e \gamma_{\nu} \Delta V / 2$ with the sign $\gamma_{\nu}=-(+)$ for $L(R)$ being $\Delta T>0$ the temperature difference and $\Delta V$ the bias voltage.

The charge current through the QD is given by $\underline{43}$

$$
J_{C}=\frac{2 e \pi}{h} A(\alpha) \Delta \int d \omega \rho(\omega)\left(f_{L}(\omega)-f_{R}(\omega),\right)
$$

where $\Delta=\Delta_{L}+\Delta_{R}$ is the total coupling of the QD to the leads with $\Delta_{\nu}=\pi \sum_{k}\left|V_{k}^{\nu}\right|^{2} \delta\left(\omega-\epsilon_{k}^{\nu}\right)$ (assumed independent of energy) and $A(\alpha)=4 \alpha /(\alpha+1)^{2}$ represent the asymmetry factor. In addition, $f_{\nu}(\omega)=$ $1 /\left(\exp \left(\frac{\omega-\mu_{\nu}}{T_{\nu}}+1\right)\right)$ is the Fermi distribution associated to the lead $\nu$, and the spectral function of the QD per spin, is given by $\rho(\omega)$.

In the following, we assume $U \rightarrow \infty$, which is a realistic limit for most molecular QDs. In fact, renormalizing the Kondo energy scale $T_{K}$ the physics discussed here is the same for finite $U$ as long as the Kondo peak (the one near the Fermi energy and of width $2 T_{K} \ll \Delta$ ) in the spectral density of states is well separated from the charge-transfer peaks at $E_{d}$ and $E_{d}+U$ and voltages and temperatures are such that the corresponding energy scales $\mathrm{eV}$ and $T$ are much smaller than the chargetransfer excitations $\left(e V, T \ll\left|E_{d}\right|, E_{d}+U\right.$ with the Fermi energy set as $\left.\epsilon_{F}=0\right)$. Since the total width of the chargetransfer peaks in the Kondo regime $-E_{d}, E_{d}+U \gg \Delta$ are $\sim 4 \Delta, \underline{44,45}$ and $E_{d}$ can be tuned in QDs so that $E_{d} \sim-U / 2$, our results are valid for systems such that $U \geq 8 \Delta$.

\section{NONCROSSING APPROXIMATION}

To calculate the spectral function of the QD, we use the non-crossing approximation (NCA) in its non- equilibrium extension ${ }^{46,47}$. The $\mathrm{NCA}$ is equivalent to a sum of an infinite series of diagrams in perturbations in $V_{k}^{\nu}, \underline{48,49}$ In the Kondo regime, it is known to reproduce correctly the relevant energy scale $T_{K}$ and its dependence on the different parameters. The out of equilibrium NCA approach is one of the standard techniques for calculating the current in mixed valence systems within different regimes of the model, and specially within the Kondo regime (KR) where the dot occupancy is near 1 . It has proved to be a very valuable tool for calculating the differential conductance through a different systems such as two-level QD's and $\mathrm{C}_{60}$ molecules displaying a quantum phase transition, or a nanoscale Si transistor 17,50,52,53 among others. It also reproduces correctly the scaling of the conductance for small bias voltage $V$ and temperature $T, \stackrel{54}{4}$ and is able to reproduce finite-energy features in systems, where the numerical renormalization group has difficulties, like the presence of a step in the conduction band $\stackrel{55}{5}$

Alternatives to NCA for non-equilibrium problems have some limitations. For example renormalized perturbation theory is limited to small $\omega, V$ and $T 33,56-58$ and the method of the equation of motion $\sqrt[59-62]{\underline{2}}$ does nor reproduce correctly the functional dependence of $T_{K}$ on $E_{d} \underline{\underline{60}, 61}$

The main limitations of the NCA for the Anderson model we consider take place for moderate positive $E_{d}$ and finite moderate $U$. For positive $E_{d}$, the impurity self energy has an unphysical positive imaginary part and as a consequence $\rho(\omega)$ presents a spurious peak at the Fermi energy. Similar spurious peaks exist for finite magnetic field $\stackrel{46}{\underline{H}}$ For finite $U$ the NCA ceases to reproduce correctly the dependence of $T_{K}$ with parameters and vertex corrections should be included ${ }^{63}-65$ Since in this work, the parameters correspond to the Kondo regime $-E_{d}, E_{d}+U \gg \Delta$ and we take $U \rightarrow \infty$, we avoid these limitations. A minor problem is that the intensity of the Kondo peak is overestimated by about $15 \%$ compared to the value expected from the Friedel sum rule $\underline{66}$

More details on the formalism and tricks that we use to solve the selff-consistent integral equations can be found in Refs. 47 and 67 .

\section{RESULTS}

Without loss of generality, we choose our unity of energy to be $\Delta=1$. We present results for $E_{d}=-4$, which corresponds to the KR. We choose a bandwidth of $2 D$ with $D=10$. We define the Kondo temperature $\left(T_{K}\right)$ as the temperature for which the equilibrium conductance is half of the corresponding one for the unitary limit, $G\left(T=T_{K}\right)=G_{0} / 2$ with $G_{0}=2 A(\alpha) e^{2} / h$. For our parameters, this leads to $T_{K}=0.0086$. Due to the universality of the model in the KR the results presented here in units of $T_{K}$ are quite general in this regime and do not depend on the particular values of $\Delta, E_{d}$ or $U$. For simplicity, we also take the Boltzmann constant and 
absolute value of the electronic charge $k_{B}=e=1$ so that The Seebeck coefficient $S$ and $V / T$ become dimensionless.

The Seebeck coefficient, $S$, and the electrical conductance, $G$, within the linear response regime, $\Delta T=$ $\Delta V \rightarrow 0$, are commonly defined in terms of equilibrium properties ${ }^{26}, S=-I_{1}(T) /\left[e T I_{0}(T)\right], G=e^{2} I_{0}(T)$ being

$$
I_{n}(T)=\frac{2 \pi}{h} A(\alpha) \Delta \int d \omega \omega^{n} \rho(\omega)\left(-f^{\prime}(\omega)\right) .
$$

Here, the asymmetry factor $\alpha$ does not modify the equilibrium spectral density due to the fact that both, the charge-transfer peak near $E_{d}$ and the Kondo one at the Fermi energy $\epsilon_{F}=0$ depend on the total coupling $\Delta=\Delta_{L}+\Delta_{R}$ and not on the ratio $\alpha=\Delta_{L} / \Delta_{R}$. While the charge-transfer peak has a width of $4 \Delta$ in the $\mathrm{KR}^{44,45}$, the width of the Kondo peak is of the order of $T_{K} \sim D \exp \left(\pi E_{d} / 2 \Delta\right)$ and its intensity is fixed by the Friedel sum rule $\rho(0) \sim 1 / \pi \Delta$.

In Fig. 2(b) we show the spectral density for two extremely different values of $\alpha$. As explained above, they are identical. Thus, the equilibrium conductance is strongly suppressed for high values of the asymmetry $\alpha$ [due to the factor $A(\alpha))$ in Eq. [2)] while the Seebeck coefficient remains unchanged due to the cancellation of $A(\alpha)$ in $I_{1}(T) / I_{0}(T)$ as it can be seen in the left panel of Fig. 2.

However, when finite values of the bias voltage are considered, non trivial effects arise, which depend on $\alpha$. The Kondo resonance in the spectral density splits in two

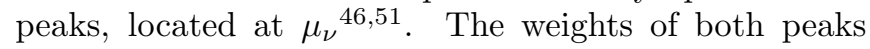
are affected by $\alpha$. While they are approximately equal in the symmetric case, $\alpha=1$, the one at $\mu_{R}\left(\mu_{L}\right)$ decreases (increases) for increasing $\alpha$. For $\alpha \gg 1$, the QD is nearly in equilibrium with the left lead and the spectral density shows the full Kondo resonance shifted to the chemical potential $\mu_{L}$, see Fig. 2(c). On the other hand, the differential conductance, $G(\Delta V)=d J_{C} / d(\Delta V)$, is an even function of the bias voltage for $\alpha=1$ due to left-right reflection symmetry but in the case of $\alpha \gg 1$ it mimics the spectral density, $G(\Delta V) \sim \frac{e^{2}}{h} \pi \Delta A(\alpha) \rho(-e \Delta V / 2)$, see inset of Fig. 2(a). Similar conclusions were drown in Refs. 24 and 44.

In what follows we focus on the analysis of nonlinear effects on the differential Seebeck coefficient and its dependence on $\alpha$. In analogy to bulk systems, when a temperature difference $\Delta T$ is applied between both sides of a $\mathrm{QD}$, keeping $J_{C}=0$, a voltage difference $\Delta V$ proportional to $\Delta T$ appears, $S=-\left.\frac{\Delta V}{\Delta T}\right|_{J_{C}=0}$. Non linear effects following the line $J_{C}=0$ have been already addressed 24,27,31,34,39. However, Dorda et al. have recently analyzed the non linear regime across the line $J_{C} \neq 0$,

$$
\mathcal{S}=-\left.\frac{d(\Delta V)}{d(\Delta T)}\right|_{J_{C}=\text { const. }}=\frac{\partial J_{C}}{\partial(\Delta T)} / \frac{\partial J_{C}}{\partial(\Delta V)}
$$

which is expected to be a measurable quantity that contributes to the better knowledge of the two decoherence

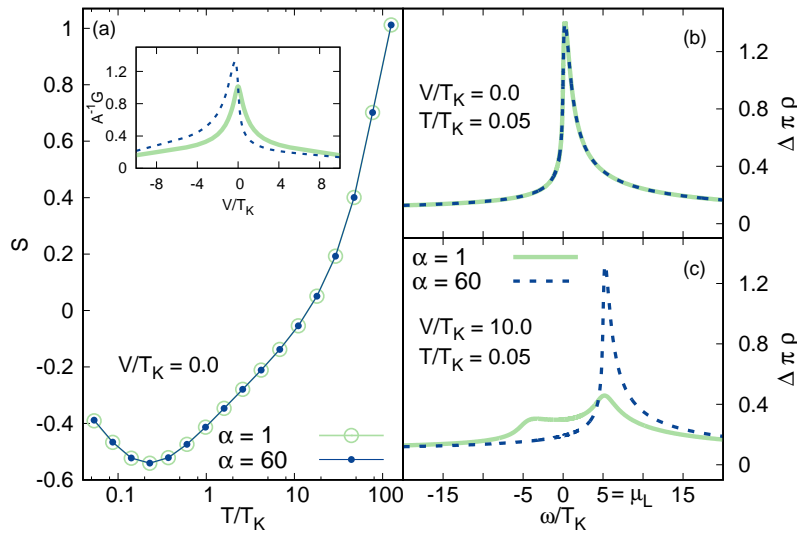

FIG. 2. (Color online) (a) Thermopower as a function of temperature for two values of the asymmetry ratio $\alpha$ at equilibrium $(\Delta V=\Delta T=0)$. The inset shows the differential conductance as a function of bias voltage for two values of $\alpha$ (1 and 60). (b) Spectral density as a function of energy at equilibrium for the same $\alpha$ as in (a). (c) Spectral density as a function of energy for $V / T_{K}=10$ for the same $\alpha$ as in (a). In all cases $T / T_{K}=0.05$.

processes generated by the bias voltage and temperature and can be important for applications in nanoscale temperature sensing 35 .

We compute numerically both $\partial J_{C} / \partial(\Delta V)$ from Eq. (2) and $\partial J_{C} / \partial(\Delta T)$ using

$$
\begin{aligned}
\frac{\partial J_{C}}{\partial(\Delta T)} & =\frac{2 e \pi}{h} A(\alpha) \Delta \int d \omega\left[\frac{1}{2 T} \rho(\omega) \sum_{\nu}\left(\omega-\mu_{\nu}\right) \frac{d f_{\nu}}{d \omega}(\omega)\right. \\
& \left.+\left(f_{L}(\omega)-f_{R}(\omega)\right) \frac{\partial \rho(\omega)}{\partial(\Delta T)}\right] .
\end{aligned}
$$

We will show that the differential thermopower at finite bias voltage as described by Eq. (4) is largely enhanced for large asymmetry between the tunnel couplings.

In Fig. 3 we show the results for the differential Seebeck coefficient at finite voltage as a function of temperature and for several values of the asymmetry factor $\alpha$. For $T \gg T_{K}, \mathcal{S}$ does not depend on $\alpha$ independently of the sign of the current. In fact, at high $T$ there is no Kondo peak in the spectral density and the electronic transport is hole-like mediated by the charge transfer peak located at $\omega \sim E_{d}<0$, that has a width of the order of $4 \Delta^{44.45}$, and is nearly not affected for the bias voltage considered in the figure, of the order of a few $T_{K}$. For finite $U$, the other charge transfer peak at $\omega \sim E_{d}+U>0$, neglected in our approach can modify the results. However, we are interested in the regime of temperatures of the order of a few times $T_{K}$ or less. For these temperatures, only the spectral density at low energies is relevant due to the small window of the Fermi functions. One can observe a significant increment of the magnitude of $\mathcal{S}$ for larger values of $\alpha$ as compared to the symmetric case. In particular for $V=-10 T_{K}$ and temperatures of the order of $T_{K}$ or below it, $|\mathcal{S}|$ is enhanced by a factor near 5 as the 


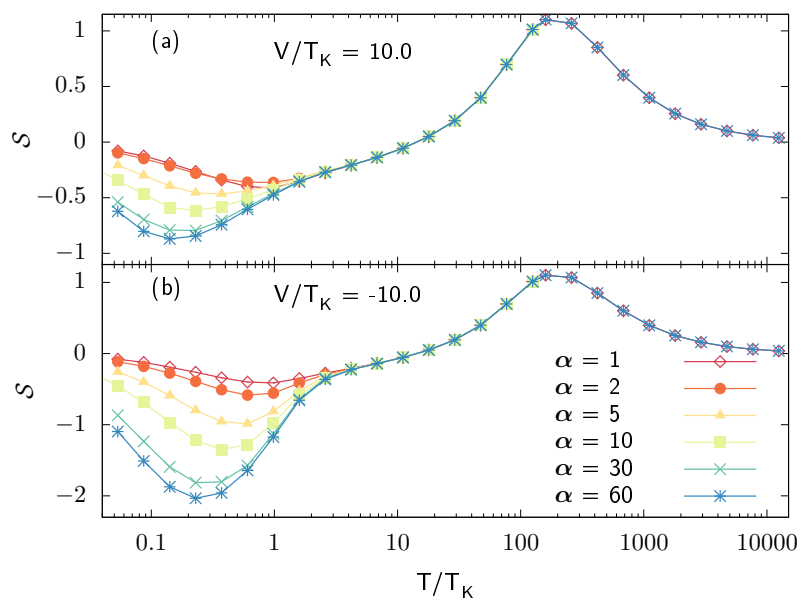

FIG. 3. (Color online) Differential thermopower as a function of temperature for several values of the asymmetry $\alpha$, (a) with $e \Delta V / T_{K}=10$ and (b) with $e \Delta V / T_{K}=-10$.

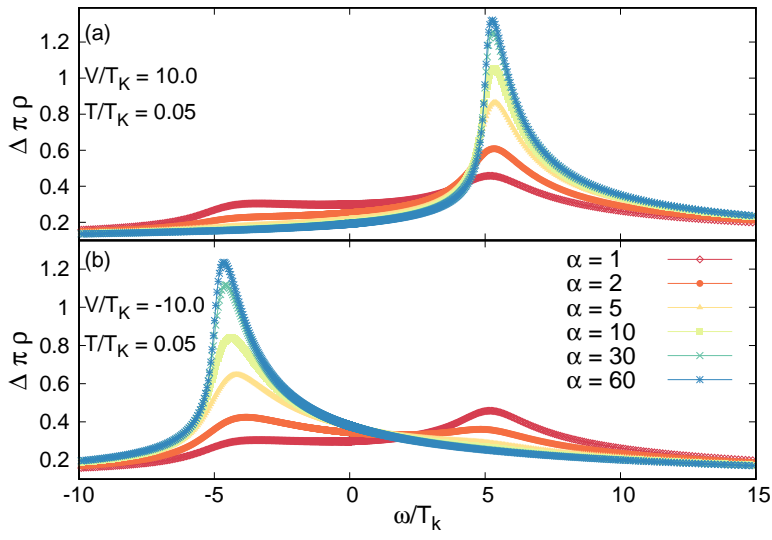

FIG. 4. (Color online) Kondo resonance in the QD spectral density under a finite bias voltage for several values of the asymmetry ratio $\alpha$. (a) Positive voltage, $\mu_{L} / T_{K}=5, T_{K}=$ 0.008. (b) Negative voltage, $\mu_{L} / T_{K}=-5, T_{K}=0.01$.

asymmetry $\alpha$ increases from 1 (symmetric case) to 60 .

\section{INTERPRETATION AND FURTHER RESULTS}

In order to understand the behavior of the thermoelectric response as $\alpha$ is varied, we analyze qualitatively the low-temperature features of the charge current in Eq. (2). As we have showed in Fig. 2, for large enough asymmetry the dot tends to be in equilibrium with the left lead. The evolution of the spectral density with $\alpha$ is explicitly shown in Fig. 4 for both signs of the applied bias voltage.

If the dot is in equilibrium with the left lead, then $\int d \omega \rho(\omega) f_{L}(\omega)$ does not change with the applied voltage. Using this assumption and standard Sommerfeld expansion $^{22}$ for the current in Eq. (2), the low temperature behavior of the differential Seebeck coefficient reads

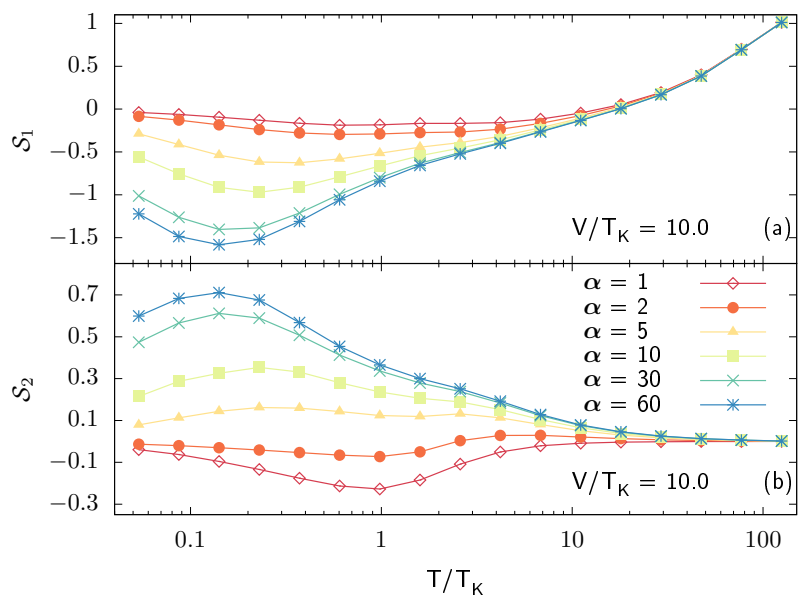

FIG. 5. (Color online) Contributions to $\mathcal{S}$ of the first and second members of Eq. (5), $\mathcal{S}_{1}$ and $\mathcal{S}_{2}$ respectively, as a function of temperature and for positive bias voltage.

as follows,

$$
\begin{aligned}
\mathcal{S}(T) & \sim\left[\rho\left(\mu_{R}\right)\right]^{-1}\left[-\frac{\pi^{2} T}{6 e} \sum_{\nu} \rho^{\prime}\left(\mu_{\nu}\right)\right. \\
& \left.+\int_{\mu_{R}}^{\mu_{L}} d \omega h(\omega)+\frac{\pi^{2} T^{2}}{6 e}\left(h^{\prime}\left(\mu_{L}\right)-h^{\prime}\left(\mu_{R}\right)\right)\right],
\end{aligned}
$$

being $h=\partial \rho / \partial \Delta T$ and $A^{\prime} \equiv d A / d \omega$. Fig. 4 demonstrates that $\sum_{\nu} \rho^{\prime}\left(\mu_{\nu}\right)$ in the first term of the right-hand side of Eq. (6) increases as the coefficient $\alpha$ does. On the other hand, the value of $\rho\left(\mu_{R}\right)$ continuously decreases when $\alpha$ is increased. Interestingly, both tendencies contribute to boost the coefficient $\mathcal{S}$.

While the temperature dependence of $\mathcal{S}$ is qualitatively the same for positive as well as negative bias voltage, as it is shown in panel (a) $(\Delta V>0)$ and (b) $(\Delta V<0)$ of Fig. 3. the different intensity between them is related to the relative sign of the different contributions in Eq. (6). We have verified that for $\alpha>5$ the magnitude $h(\omega)$ and $h^{\prime}(\omega)$ are positive within the relevant energy range, and therefore, the second term of Eq. (6) is also positive.

In Fig. 5 we separately show for a positive value of voltage, the first and second contributions to $\mathcal{S}$ from Eq. (5), namely $\mathcal{S}_{1}$ and $\mathcal{S}_{2}$ respectively. While the total magnitude of $\mathcal{S}$ is still larger for the asymmetric case, it is clear that both contributions partially compensate each other. However, for negative voltage (not shown) both terms have the same negative sign and boost even further the magnitude of $\mathcal{S}$. The chain in sign is clearly due to the third (last) term in Eq. (6). Therefore, larger intensity of the differential response is obtained for $\mu_{L}<0$, that is when the current flows from the less coupled (right) lead to the other one. This observation can be used to determine experimentally, in a very simple way, to what side ( $L$ or $R$ ) the molecule or QD is closer or more coupled.

In Fig. 6 we show the behavior of the thermopower at two different temperatures and for several values of the asymmetry factor $\alpha$ as a function of the applied bias 


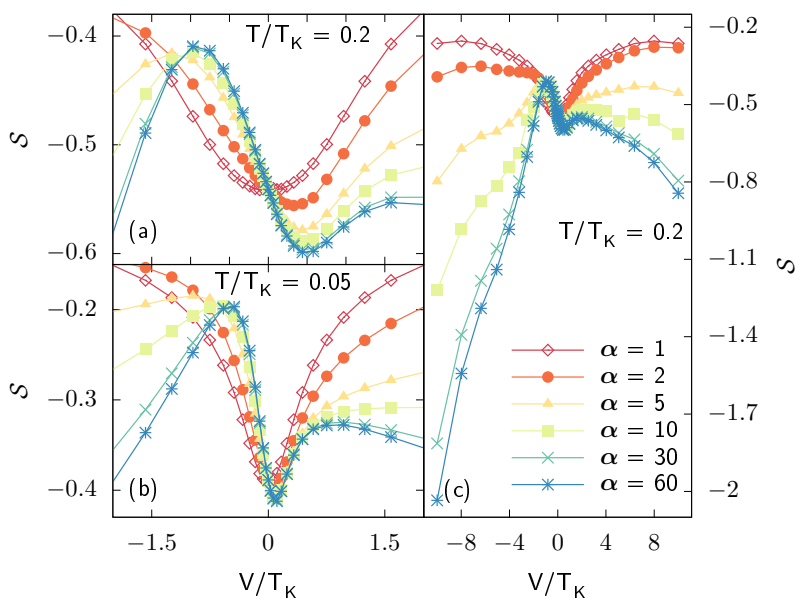

FIG. 6. (Color online) Differential thermopower as a function of the bias voltage in units of $T_{K}$ for several values of the asymmetry ratio $\alpha$ and at low temperature.

voltage. As a first observation, for the bias voltage considered here, $|e \Delta V| \sim T_{K}$, the sign of $\mathcal{S}$ is negative in agreement with Fig. 3 for $T<T_{K}$, due to the electronic character of the transport.

In the case of symmetric couplings, $\alpha=1, \mathcal{S}$ as defined in Eq. (4) is characterized by an even function of the applied bias voltage $e^{68}$. The minimum at $e \Delta V=0$ is due to finite temperature effects. From Eq. (5) $\mathcal{S}$ is expected to vanish in the zero temperature limit. This tendency can be observed by a comparison of the minimum value of $\mathcal{S}$ for the two selected temperatures in Fig. 6. $T=$ $0.2 T_{K}$ and $T=0.05 T_{K}$. On the other hand, for large values of $e \Delta V$, the argument of Eq. (5) tends to be an odd function within the relevant range of energies. Note that the later can be written as $[\rho(\omega+e \Delta V / 2)+$ $\rho(\omega-e \Delta V / 2)] \omega f^{\prime}(\omega)$ in case of the first contribution (See Fig. 2(c)). Regarding the second one, we note that $h_{\nu}(\omega)$ has structure at both $\mu_{\nu}$ and therefore the product $\left(f_{L}(\omega)-f_{R}(\omega)\right) h(\omega)$ approaches an odd function of the energy.

On the other hand, the case of $\alpha \gg 1$ is quite different. While the behavior of $\mathcal{S}$ for $|e \Delta V| / T_{K} \ll 1$ is still governed by temperature effects, its dependence on $e \Delta V$ has no parity. The situation of finite values of the bias voltage, particularly at values of a few times $T_{K}$, is more involved. As soon as $|e \Delta V|$ reaches $T_{K}$, the absolute value of $\mathcal{S}$ is largely increased. Once again, the mechanism behind this behavior is the displacement of $\rho(\omega)$ towards $\omega \sim \mu_{L}$. The same mechanism that explained the behavior of $\mathcal{S}$ in Fig. 3 applies in the case of the voltage dependence. Large values of $\mathcal{S}$ are obtained for large enough asymmetry. In particular, the increment is even larger when negative voltage is considered as explained before. Note that for negative $V$ an increase in $|\mathcal{S}|$ for more than an order of magnitude is obtained as $\alpha$ increases from 1 to 60 .

\section{CONCLUSIONS}

In summary, we have investigated theoretically the differential response of the electric current at finite bias voltage, when an infinitesimal gradient of temperature is applied to a system of a molecular or semiconducting quantum dot coupled asymmetrically to two conducting leads. We have concentrated on the electronic contribution to the differential Seebeck coefficient $\mathcal{S}$, leaving aside phonon contributions.

We show that $\mathcal{S}$ is strongly enhanced for a large asymmetry between the tunnel couplings. In particular, we find an enhancement of $\mathcal{S}$ by an order of magnitude at temperatures of the order of a fraction of the Kondo temperature $T_{K}$ and bias voltage $\mathrm{eV}$ of the order of several times $k_{B} T_{K}$. This becomes relevant for the standard cryogenic experimental conditions, for which the Kondo effect emerges. In Section $\mathrm{V}$ we have provided an explanation of the non-trivial mechanism behind our findings and we believe that it could be useful for experimental purposes. Our findings can also be important for nanoscale temperature sensing.

Although the calculation of the figure of merit $\mathrm{ZT}$ is beyond the scope of this work, our results suggest that molecular quantum dots, for which large tunneling asymmetries are expected, or semiconducting quantum dots in which a large asymmetry can be tuned easily, would be the most efficient quantum machines operating as both, heat-engines or refrigerators.

\section{ACKNOWLEDGMENTS}

We are partially supported by CONICET, Argentina. A. A. A, was sponsored by PIP 112-201101-00832 of CONICET and PICT 2013-1045 of the ANPCyT.
1 G. Benient, G. Casati, K. Saito, R. S. Whitney, Fundamental aspects of steady-state conversion of heat to work at the nanoscale, Phys. Rep. 694, 1 (2017).

2 Kondo effect in a single-electron transistor, D. GoldhaberGordon, H. Shtrikman, D. Mahalu, D. Abusch-Magder, U. Meirav, and M. A. Kastner, Nature 391, 156 (1998).

3 A Tunable Kondo Effect in Quantum Dots, S. M. Cronenwett, T. H. Oosterkamp, and L. P. Kouwenhoven, Science
281, 540 (1998)

4 From the Kondo Regime to the Mixed-Valence Regime in a Single-Electron Transistor, D. Goldhaber-Gordon, J. Göres, M. A. Kastner, H. Shtrikman, D. Mahalu, and U. Meirav, Phys. Rev. Lett. 81, 5225 (1998).

5 The Kondo Effect in the Unitary Limit, W.G. van der Wiel, S. de Franceschi, T. Fujisawa, J.M. Elzerman, S. Tarucha, and L.P. Kowenhoven, Science 289, 2105 (2000). 
6 Universal Scaling in Nonequilibrium Transport through a Single Channel Kondo Dot, M. Grobis, I. G. Rau, R. M. Potok, H. Shtrikman, and D. Goldhaber-Gordon, Phys. Rev. Lett. 100, 246601 (2008).

7 Spin- $\frac{1}{2}$ Kondo effect in an InAs nanowire quantum dot: Unitary limit, conductance scaling, and Zeeman splitting, A. V. Kretinin, H. Shtrikman, D. Goldhaber-Gordon, M. Hanl, A. Weichselbaum, J. von Delft, T. Costi, and D. Mahalu, Phys. Rev. B 84, 245316 (2011).

8 Pseudospin-Resolved Transport Spectroscopy of the Kondo Effect in a Double Quantum Dot, S. Amasha, A. J. Keller, I. G. Rau, A. Carmi, J. A. Katine, H. Shtrikman, Y. Oreg, and D. Goldhaber-Gordon, Phys. Rev. Lett. 110, 046604 (2013).

9 Kondo resonance in a single-molecule transistor, W. Liang, M. P. Shores, M. Bockrath, J. R. Long, and H. Park, Nature 417,725 (2002).

10 Single-electron transistor of a single organic molecule with access to several redox states, S. Kubatkin, A. Danilov, M. Hjort, J. Cornil, J. L, Brédas, N. Stuhr-Hansen, P. Hedegård, and Th. Bjørnholm, Nature 425, 699 (2003).

11 Kondo Resonances and Anomalous Gate Dependence in the Electrical Conductivity of Single-Molecule Transistors, L. H. Yu, Z. K. Keane, J. W. Ciszek, L. Cheng, J. M. Tour, T. Baruah, M. R. Pederson, and D. Natelson, Phys. Rev. Lett. 95, 256803 (2005).

12 Berry-Phase Oscillations of the Kondo Effect in SingleMolecule Magnets, M. N. Leuenberger and E. R. Mucciolo, Phys. Rev. Lett. 97, 126601 (2006).

13 Tuning the Kondo Effect with a Mechanically Controllable Break Junction, J. J. Parks, A. R. Champagne, G. R. Hutchison, S. Flores-Torres, H. D. Abruña, and D. C. Ralph, Phys. Rev. Lett. 99, 026601 (2007).

14 Quantum phase transition in a single-molecule quantum dot, N. Roch, S. Florens, V. Bouchiat, W. Wernsdorfer, and F. Balestro, Nature 453, 633 (2008).

15 Universal scaling of nonequilibrium transport in the Kondo regime of single molecule devices, G. D. Scott, Z. K. Keane, J. W. Ciszek, J. M. Tour, and D. Natelson, Phys. Rev. B 79, 165413 (2009).

16 Mechanical Control of Spin States in Spin-1 Molecules and the Underscreened Kondo Effect, J. J. Parks, A. R. Champagne, T. A. Costi, W. W. Shum, A. N. Pasupathy, E. Neuscamman, S. Flores-Torres, P. S. Cornaglia, A. A. Aligia, C. A. Balseiro, G. K.-L. Chan, H. D. Abruñ a, and D. C. Ralph, Science 328, 1370 (2010).

17 Universal transport signatures in two-electron molecular quantum dots: gate-tunable Hund's rule, underscreened Kondo effect and quantum phase transitions, S. Florens, A, Freyn, N. Roch, W. Wernsdorfer, F. Balestro, P. RouraBas and A. A. Aligia, J. Phys. Condens. Matter 23, 243202 (2011); references therein.

18 Electronic read-out of a single nuclear spin using a molecular spin transistor, R. Vincent, S. Klyatskaya, M. Ruben, W. Wernsdorfer, and F. Balestro, Nature (London) 488, 357 (2012).

19 Perspective: Thermal and thermoelectric transport in molecular junctions, L. Cui, R. Miao, C. Jiang, E. Meyhofer, and P, Reddy, The Journal of Chemical Physics,146, 092201(2017).

20 Single Molecule Conductance, Thermopower, and Transition Voltage, S. Guo, G. Zhou, and N. Tao, Nano Lett. 13, 4326 (2013).
21 Length-dependent thermopower determination of amineterminated oligophenyl single molecular junctions formed with Ag electrodes, D. Kim, P. S. Yoo, and T. Kim, J. Korean Phys. Soc. 66, 602 (2015).

22 Thermopower measurement in molecular junctions, $\mathrm{L}$. Rincón-García, C. Evangeli, G. Rubio-Bollinger, and N. Agraït, Chem. Soc. Rev., 45, 4285 (2016); references therein.

23 The best thermoelectric, G. D. Mahan and J. O. Sofo; Proc. Natl. Acad. Sci. USA 93, 7436 (1996).

24 Thermoelectric phenomena in quantum dot asymmetrically coupled to external leads, M. Krawiec, and K. I. Wysokiński, Phys. Rev. B 75, 155330 (2007).

25 Thermal transport in one-dimensional spin heterostructures, L. Arrachea, G. S. Lozano, and A. A. Aligia Phys. Rev. B 80, 014425 (2009).

26 T. A. Costi and V. Zlatić, Phys. Rev. B 81, 235127 (2010).

27 Nonlinear thermoelectric properties of molecular junctions with vibrational coupling, M. Leijnse, M. R. Wegewijs, and K. Flensberg, Phys. Rev. B 82, 045412 (2010).

28 Thermoelectric efficiency at maximum power in lowdimensional systems, N. Nakpathomkun, H. Q. Xu, and H. Linke; Phys. Rev. B 82, 235428 (2010).

29 Mechanism for large thermoelectric power in molecular quantum dots described by the negative- $U$ Anderson model, S. Andergassen, T. A. Costi, and V. Zlatić; Phys. Rev. B 84, 241007(R) (2011).

30 Enhance thermopower under a time-dependent gate voltage, A. Crépieux, F. Simkovic, B. Cambon, and F. Michelini; Phys. Rev. B 83, 153417 (2011).

31 Kondo physics and orbital degeneracy interact to boost thermoelectrics on the nanoscale, J. Azema, A.-M. Daré, S. Schäfer, and P. Lombardo, Phys. Rev. B 86, 075303 (2012).

32 Thermopower of an $S U(4)$ Kondo resonance under an SU(2) symmetry-breaking field, P. Roura-Bas, L. Tosi, A. A. Aligia, and P. S. Cornaglia, Phys. Rev. B 86, 165106 (2012).

33 Nonequilibrium self-energies, $\mathrm{Ng}$ approach, and heat current of a nanodevice for small bias voltage and temperature, A. A. Aligia Phys. Rev. B 89, 125405 (2014); references therein.

34 Conditions for requiring nonlinear thermoelectric transport theory in nanodevices, J. Azema, P. Lombardo, and A.-M. Daré, Phys. Rev. B 90, 205437 (2014).

35 Thermoelectric response of a correlated impurity in the nonequilibrium Kondo regime, A. Dorda, M. Ganahl, S. Andergassen, W. von der Linden, and E. Arrigoni, Phys. Rev. B 94, 245125 (2016).

36 Thermoelectric properties of an interacting quantum dot based heat engine, P. A. Erdman, F. Mazza, R. Bosisio, G. Benenti, R. Fazio, and F. Taddei; Phys. Rev. B 95, 245432 (2017).

37 Thermal conductance and thermoelectric figure of merit of C60 -based single-molecule junctions: Electrons, phonons, and photons, J. C. Klöckner, R. Siebler, J. C. Cuevas and F. Pauly Phys. Rev. B 95, 245404 (2017).

38 Fate of the spin-1/2 Kondo effect in the presence of temperature gradients, M. A. Sierra, R. López, and D. Sánchez, Phys. Rev. B 96, 085416 (2017).

39 Enhance Thermoelectric Performance Using Nonlinear Transport Effects, Jian-Hua Jiang, and Y. Imry; Phys. Rev. App. 7, 064001 (2017). 
40 Enhanced thermoelectric response in the fractional quantum Hall effect, P. Roura-Bas, L. Arrachea, and E. Fradkin, Phys. Rev. B 97, 081104(R) (2018).

41 J. Könemann, B. Kubala, J. König, and R. J. Haug, Phys. Rev. B 73, 033313 (2006).

42 Electron-Vibration Interaction in the Presence of a Switchable Kondo Resonance Realized in a Molecular Junction, D. Rakhmilevitch, R. Korytár, A. Bagrets, F. Evers, and O. Tal, Phys. Rev. Lett. 113, 236603 (2014).

43 Y. Meir and N. S. Wingreen, Phys. Rev. Lett. 68, 2512 (1992).

44 Impact of capacitance and tunneling asymmetries on Coulomb blockade edges and Kondo peaks in nonequilibrium transport through molecular quantum dots, A. A. Aligia, P. Roura-Bas, and S. Florens, Phys. Rev. B 92, 035404 (2015).

45 Width of the charge-transfer peak in the $S U(N)$ impurity Anderson model and its relevance to nonequilibrium transport, J. Fernández, F. Lisandrini, P. Roura-Bas, C. Gazza, and A. A. Aligia, Phys. Rev. B 97, 045144 (2018).

46 Anderson model out of equilibrium: Noncrossingapproximation approach to transport through a quantum dot, N. S. Wingreen and Y. Meir, Phys. Rev. B 49, 11040 (1994).

47 Nonequilibrium transport through magnetic vibrating molecules, P. Roura-Bas, L. Tosi and A. A. Aligia, Phys. Rev. B 87, 195136 (2013); references therein.

48 Review of techniques in the large- $N$ expansion for dilute magnetic alloys, N.E. Bickers, Rev. of Mod. Phys. 59, 845 (1987).

49 Fermi and Non-Fermi Liquid Behavior in Quantum Impurity Systems: Conserving Slave Boson Theory, J. Kroha and P. Wölfle, Acta Phys. Pol. B 29, 3781 (1998).

50 Nonequilibrium transport through a singlet-triplet Anderson impurity, P. Roura-Bas, A. A. Aligia Phys. Rev. B 80, 035308 (2009).

51 Nonequilibrium dynamics of a singlet-triplet Anderson impurity near the quantum phase transition, P. Roura Bas, A. A. Aligia; J. Phys.: Condens. Matter 22, 025602 (2010).

52 Orbital Kondo spectroscopy in a double quantum dot system, L. Tosi, P. Roura-Bas and A. A. Aligia, Phys. Rev. B 88, 235427 (2013).

53 Magnetic-Field Probing of an SU(4) Kondo Resonance in a Single-Atom Transistor, G. C. Tettamanzi, J. Verduijn, G. P. Lansbergen, M. Blaauboer, M. J. Calderón, R. Aguado, and S. Rogge, Phys. Rev. Lett. 108, 046803 (2012).

54 Universal scaling in transport out of equilibrium through a single quantum dot using the noncrossing approximation, P. Roura-Bas, Phys. Rev. B 81, 155327 (2010).

${ }^{55}$ Kondo temperature when the Fermi level is near a step in the conduction density of states, J. Fernández, A. A. Aligia, P. Roura-Bas, and J. A. Andrade, Phys. Rev. B 95, 045143
(2017).

56 Non-equilibrium differential conductance through a quantum dot in a magnetic field, A. C. Hewson, J. Bauer, and A. Oguri, J. Phys.: Condens. Matter 17, 5413 (2005), and references therein.

57 Higher-order Fermi-liquid corrections for an Anderson impurity away from half filling: Nonequilibrium transport, A. Oguri and A. C. Hewson, Phys. Rev. B 97, 035435 (2018).

58 Leading temperature dependence of the conductance in Kondo-correlated quantum dots, A. A. Aligia, J. Phys. Condens. Matter xx, XX (in press) (2018), and references therein.

59 Spin fluctuation effects on the conductance through a single Pd atom contact, M. A. Romero, S C Gómez-Carrillo, P. G, Bolcatto, and E. C. Goldberg, J. Phys. Condens. Matter 21, 215602 (2009).

60 Anderson model out of equilibrium: Decoherence effects in transport through a quantum dot, R. Van Roermund, S. Y. Shiau, and M. Lavagna Phys. Rev. B 81, 165115 (2010).

61 Effective treatment of charge and spin fluctuations in dynamical and static atom-surface interactions, M. A. Romero, F. Flores, and E. C. Goldberg, Phys. Rev. B 80, 235427 (2009).

62 Emission Noise in an Interacting Quantum Dot: Role of Inelastic Scattering and Asymmetric Coupling to the Reservoirs, A. Crépieux, S. Sahoo, T.Q. Duong, R. Zamoum, and M. Lavagna, Phys. Rev. Lett. 120, 107702 (2018).

63 The Anderson model with finite Coulomb repulsion, Th. Pruschke and N. Grewe, Z. Phys. B Condensed Matter 74, 439 (1989).

64 Anderson impurity model at finite Coulomb interaction U: Generalized noncrossing approximation, K. Haule, S. Kirchner, J. Kroha, and P. Wölfle, Phys. Rev. B 64, 155111 (2001).

65 Effects of vertex corrections on diagrammatic approximations applied to the study of transport through a quantum dot, L. Tosi, P. Roura-Bas, A. M. Llois, and L. O. Manuel, Phys. Rev. B 83, 073301 (2011).

66 Transition between SU(4) and SU(2) Kondo effect, L. Tosi, P. Roura-Bas, and A. A. Aligia Physica B 407, 3259 (2012).

67 Non-equilibrium conductance through a benzene molecule in the Kondo regime, L. Tosi, P. Roura-Bas, and A. A. Aligia, J. Phys. Condens. Matter 24, 365301 (2012).

${ }^{68}$ In the case of $\alpha=1, \rho(\omega)$ is invariant under $\Delta V \rightarrow-\Delta V$ (and as consequence $\partial J_{C} / \partial(\Delta T)$ ), see Fig. 3. The same applies to $\sum_{\nu}\left(\omega-\mu_{\nu}\right) \frac{d f_{\nu}}{d \omega}(\omega)$. Therefore, the current in Eq. (2) is and odd function of the bias voltage while $G(\Delta V)$ is an even one. Furthermore, both contributions of Eq. (5) are invariants under $\Delta V \rightarrow-\Delta V$. 